\title{
"Warning!": from physics to an interdisciplinary project to discuss with students about the big planetary threats
}

\author{
Franco Cervelli, ${ }^{a}$ Sandra Leone ${ }^{a, *}$ and Enrico Mazzoni ${ }^{a}$ \\ ${ }^{a}$ INFN Sezione di Pisa, \\ Largo Bruno Pontecorvo 3, Pisa, Italy \\ E-mail: franco.cervelli@pi.infn.it, sandra.leone@pi.infn.it, \\ enrico.mazzoni@pi.infn.it
}

\begin{abstract}
The project "Warning! The big planetary threats: knowing them to defend ourselves" aimed to develop interdisciplinary educational paths on the themes of environmental fragility and dangerousness, addressing a wide audience but with a specific focus on young people. The initiative consisted of 5 debates were scientists presented an in-depth scientific analysis of so-called natural disasters, i.e. phenomena and consequences related to climate change, major pandemics, endogenous events (i.e. volcanoes and earthquakes), asteroid and space debris impact on earth surface and finally oceans pollution. "Being ready" was the initiative target, to be reached thanks to the adoption of responsible and scientifically sound behaviors, overcoming a culture dominated by the "here and now". An interdisciplinary approach was used stressing the importance of the scientific method to face complex problems. The various events took place virtually, allowing the participation of more than 3000 high school students. "Warning" represented a useful educational support for students and teachers, who were able to attend the events in a "virtual classroom", and integrate topics covered in school programs.
\end{abstract}

\footnotetext{
*** The European Physical Society Conference on High Energy Physics (EPS-HEP2021), ***

*** 26-30 July $2021 * * *$

*** Online conference, jointly organized by Universität Hamburg and the research center DESY ***
}

\footnotetext{
${ }^{*}$ Speaker
} 


\section{Introduction}

Pandemics (like Covid19, that caused a global crisis all over the world in the last two years) are not the only source of risks at planetary level. It's well known that $20 \%$ of the Earth is permanently vulnerable to highly dangerous natural hazards: more than 30\% of European population lives in dangerous areas and large geographic regions are exposed to multiple types of serious natural risks. Earthquakes, volcanic eruptions, hurricanes, climatic changes and even interactions with asteroids can jeopardize our lives. All these phenomena have complex features: the unpredictability or difficult predictability of their development, the quantification of the risks, the possible dangerous interconnections among them, the diversity of their impact according to the social, economic and cultural status of the involved geographical areas. The real world is made up of complex things and the complexity manifests itself in the incapacity of any formalism to capture all of its properties: the scientific method is the primary tool to deal with complexity.

To draw the attention of new generations to events potentially threatening the whole mankind, Pisa Foundation and the Italian Institute for Nuclear Physics (INFN) launched the initiative called "Warning: the great planetary threats. Knowing them to defend ourselves", which took place between December 2020 and May 2021. The aim was to help participants to achieve awareness of the importance of preparing to "be ready", thanks to the adoption of responsible and scientifically sound behaviors, addressing the limits of a culture dominated by the "here and now", as it is the modern society, little motivated to tackle long-term problems.

Interdisciplinary approach, importance of cross knowledge and, above all, education on complexity, were the main features of "Warning". Instead of focusing on a specific aspect, interdisciplinarity allows to integrate different topics with real life problems. Strategies peculiar to physics were the "fil rouge" accompanying the participants in this journey across many fields of science. Indeed, interdisciplinarity promotes epistemological approaches (what can be known about it?) and methodological insights (how can it be found out?). It is essential to review and compare future risks using a common set of metrics, and to assess how the size and nature of risks are evolving. Integration of knowledge, at the cross-roads of different scientific disciplines, helps to find sustainable solutions in a wide range of hazardous contexts, so reducing risks and mitigating their consequences.

"Warning" was conceived as a knowledge dissemination project, addressing the general public at large, but with a specific focus on young people. The initiative was articulated in five events, each devoted to improve the scientific knowledge of potentially dangerous natural hazards and to encourage precautionary and defensive behaviors. Each topic of "Warning" was faced from the perspective of interdisciplinarity and complexity. The importance of internationally supportive initiatives to address these dangers was outlined as well.

\section{The organization of the events}

The partners in this project are the Italian National Institute for Nuclear Physics (INFN) and Fondazione Pisa. INFN is the research agency dedicated in Italy to the study of the fundamental constituents of matter. INFN mission includes the transfer of acquired knowledge to society, intended as both technological transfer and diffusion of the scientific culture, promoting dialogue 
with citizens. Fondazione Pisa has its origins from a banking foundation, and it's active in Pisa and surrounding areas in the fields of cultural heritage, scientific research and philanthropy. Palazzo Blu is the center of temporary exhibitions and cultural activities managed by Fondazione Pisa. Two renowned speakers with complementary expertise were invited for each event [1], [2], [3], [4], [5].

\section{Methodology}

Due to the imminent risk of the pandemic lockdown all events took place virtually. More than 3000 students from 25 high schools all around Tuscany participated, through the mediation of their teachers. To encourage the conscious and direct participation of students in the debate, some explanatory material in digital form was made available before the meetings. Students were asked to prepare and present their questions to the speakers in advance. On average we collected 600 questions for each seminar, therefore an accurate procedure of classification and selection was necessary. Each event was not just a seminar, but the end-point of a long educational path: a significant part of the seminars was devoted to answering student questions. "Warning" had a dedicated website, used for the dissemination of the project's activities, with individual sections for each of the five topics. Each event was streamed online on "Warning" web site and social media. The seminar's recording was made available from the web site after the event. On average each event was followed online by 700 registered participants and obtained offline from 3 to 9 thousand visualizations. Many of the participating groups of students produced a final script in which they presented a personal elaboration on some of the themes proposed during the project.

\section{The topics}

The five events were devoted to alarms from the atmosphere, major biological hazards, geological hazards, risks from the space and the relationship between oceans and climate changes. We cannot list here all the interesting aspects that were covered during the seminars: we give just a glimpse at the five topics.

\subsection{Alarms from Atmosphere}

This theme was not only debated to show how and why human activities lead large quantities of Green House Gases (GHG) into the atmosphere, causing the growth of the greenhouse effect [1]. Historical experimental data were also reported, showing that the air average temperature increased by more than 1 degree since 1860 until today, and that the global warming in the 20th century was the highest of the last millennium. Besides its serious immediate impact on the environment and people, climate change is one of the biggest threats to economic stability. Heatwaves, hurricanes, cyclones and typhoons devastate whole countries, leaving millions of people in absolute poverty. There is a strong consensus in the scientific community on the urgency to reduce GHG emissions significantly.

\subsection{Major biological hazards}

The event had its focus on Covid-19. Being well informed about this virus, i.e the disease it causes and how it spreads, was demonstrated to guarantee the best way to prevent and slow down the transmission. Vaccines were described in details and recommended [2]. 
An overview was given of how in history pandemics changed the destiny of entire populations, and how the evolution of human habits influenced the spread of diseases. It was outlined that, over the centuries, implementation of public health measures such as isolation, quarantine and border control to contain the spread of infectious diseases and maintain the structure of the society was a common practice (not very different from what is happening today).

\subsection{Restless soil}

The aim of this seminar was to present the study of volcanic and seismic risk analyzing various aspects of this subject [3]. The issue of risk communication and the importance of the adoption of measures to contain risks were discussed. In was pointed out that, while geophysics provides the tools to understand the dynamic of seismic and volcanic events, only an integrated analysis including a good knoledge of history, physical geography, social sciences and economics provides a complete overview of these hazards.

The interaction between humans and the volcanic-seismic environments is multifaceted and complex. Many people choose - rather than being forced - to live with volcanic-seismic hazards. They are not only exposed to its negative consequences, but also enjoy benefits and opportunities of physical, socio-cultural and also spiritual nature that arise within the human-volcanic system. This calls for new approaches to population management, preparedness, and proactive initiatives.

\subsection{Astonishments and hidden dangers from Space}

Since prehistoric times unexpected astronomical bodies, like long-period comets, aroused interest or even dread but their true danger was not understood: the craters on the Earth and the Moon were generally thought to be of volcanic origin and not made by impacts [4]. Only in the eighties of the last century it was suggested that the dinosaurs had been extinguished as the result of a large object hitting the Earth 65 million years ago. While we are becoming incredibly efficient at spotting Near-Earth Objects (NEO), we still have no tested solution about comets or asteroids that might be on a collision path with the Earth. In front of a NEO planetary risk, essential prerequisites to mitigation are the discovery of the NEO well in advance of possible impact, but also the possibility to achieve a collaboration at planetary level to look into the practical possibilities of countermeasures.

\subsection{Climate and Oceans: sinister changes}

The ocean makes up $71 \%$ of the planet and provides many services to human communities, from mitigating weather extremes to generating the oxygen we breathe, from producing the food we eat to storing the excess carbon dioxide we generate [5].. The consequences of ocean warming are already observable: the ocean is being disproportionately affected by carbon dioxide increase caused by human activities. This causes changes in water temperature, ocean acidification and deoxygenation, leading to changes in oceanic circulation and chemistry, rising sea levels and increased storm intensity.

Weakened or even lost coastal and marine ecosystems increase human vulnerability in the face of climate change and undermine the ability of countries to implement climate change adaptation and disaster risk reduction measures. Sustainable management, conservation and restoration are vital to support the continued provision of ecosystem services on which people depend. 


\section{The survey}

After the end of the seminar series we proposed a questionnaire to the teachers that coordinated the students' participation in the project, to assess if the initiative had been favorably received. The general feedback was very positive. Some perplexities emerged only about the level of direct student involvement, which was considered not completely satisfactory. This aspect is something we are working on, in view of a possible continuation of the project.

\section{Conclusion}

The scientific method and multidisciplinary approach represent the best tools we have to face complex problems. "Warning" aimed to raise young people's awareness on the planetary hazards encouraging a scientific discussion about them. The project represented a useful educational support for students and teachers, who were able to attend the events in a "virtual classroom", and integrate topics covered in school programs. The positive response, in terms of number of participants and expression of appreciation, exceeded our expectations. In consideration of this, we are preparing for a second edition, in which we plan to investigate the man-made risks that mankind can potentially face in a near future.

\section{Acknowledgements}

The authors would like to thank Fondazione Pisa and Palazzo Blu for supporting the project and in particular: Cosimo Bracci Torsi for believing in this project since the very beginning and his continuous support; Dario Moretta for coordinating the school participation and managing the question and write up collection; Maria Chiara Favilla for the general coordination and the administrative support; Susanna Bagnoli for the press releases and Mirko Delcaldo for the web site design and maintenance.

\section{References}

[1] Roberto Buizza and Francesco Lamperti (Scuola Universitaria Superiore Sant'Anna, Pisa).

[2] Francesco Menichetti (Azienda Ospedaliera Univesitaria di Pisa) and Paolo Malanima (Università di Catanzaro).

[3] Carlo Meletti (Istituto Nazionale di Geofisica e Vulcanologia (INGV), Pisa) and Viviana Castelli (INGV, Bologna).

[4] Alessandra Celletti (Universita“ di Roma Tor Vergata); Laura Faggioli and Ramona Cennamo (NEO Coordination Centre, Frascati - Rome).

[5] Elisabetta Erba (Università di Milano) and Carlo Barbante (Università di Venezia and CNR). 